\title{
BMJ Open Incidence and risk factors of delirium after percutaneous coronary intervention in individuals hospitalised for acute myocardial infarction: protocol for a systematic review and meta- analysis
}

\author{
Kaizhuang Huang (D) , ${ }^{1}$ Jiaying Lu, ${ }^{2}$ Yaoli Zhu, ${ }^{1}$ Tao Cheng, ${ }^{1}$ Dahao Du, ${ }^{1}$ \\ Xueqin Qian, ${ }^{1}$ Haiyan Pan, ${ }^{1}$ Xiaohua Wang, ${ }^{1}$ Hong Yang, ${ }^{1}$ Shaofei Lou ${ }^{1}$
}

To cite: Huang K, Lu J, Zhu Y, et al. Incidence and risk factors of delirium after percutaneous coronary intervention in individuals hospitalised for acute myocardial infarction: protocol for a systematic review and meta-analysis. BMJ Open 2020;10:e044564. doi:10.1136/ bmjopen-2020-044564

- Prepublication history and additional materials for this paper is available online. To view these files, please visit the journal online (http://dx.doi. org/10.1136/bmjopen-2020044564).

Received 06 September 2020 Revised 22 November 2020 Accepted 09 December 2020

Check for updates

(c) Author(s) (or their employer(s)) 2020. Re-use permitted under CC BY-NC. No commercial re-use. See rights and permissions. Published by BMJ.

${ }^{1}$ Intensive Care Unit, The Fifth Affiliated Hospital, Sun Yat-Sen University, Zhuhai 519000, China

${ }^{2}$ Department of Gastroenterology, The Fifth Affiliated Hospital, Sun Yat-Sen University, Zhuhai 519000, China

Correspondence to

Dr Shaofei Lou;

Ioushaofei0459@sina.com

\section{ABSTRACT}

Introduction Delirium in the postoperative period is a wide-reaching problem that affects important clinical outcomes. The incidence and risk factors of delirium in individuals with acute myocardial infarction (AMI) after primary percutaneous coronary intervention $(\mathrm{PCl})$ has not been completely determined and no relevant systematic review and meta-analysis of incidence or risk factors exists. Hence, we aim to conduct a systematic review and meta-analysis to ascertain the incidence and risk factors of delirium among AMI patients undergoing $\mathrm{PCl}$.

Methods and analyses We will undertake a comprehensive literature search among PubMed, EMBASE, Cochrane Library, PsycINF0, CINAHL and Google Scholar from their inception to the search date. Prospective cohort and cross-sectional studies that described the incidence or at least one risk factor of delirium will be eligible for inclusion. The primary outcome will be the incidence of postoperative delirium. The quality of included studies will be assessed using a risk of bias tool for prevalence studies and the Cochrane guidelines. Heterogeneity of the estimates across studies will be assessed. Incidence and risk factors associated with delirium will be extracted. Incidence data will be pooled. Each risk factor reported in the included studies will be recorded together with its statistical significance; narrative and meta-analytical approaches will be employed. The systematic review and meta-analysis will be presented according to the Preferred Reporting Items for Systematic Reviews and Meta-Analyses.

Ethics and dissemination This proposed systematic review and meta-analysis is based on published data, and thus there is no requirement for ethics approval. The study will provide an up to date and accurate incidence and risk factors of delirium after $\mathrm{PCl}$ among patients with AMI, which is necessary for future research in this area. The findings of this study will be disseminated through publication in a peer-reviewed journal. PROSPERO registration number CRD42020184388.
Strengths and limitations of this study

- This study will provide an up to date and accurate incidence of delirium after primary percutaneous coronary intervention among patients with acute myocardial infarction (AMI).

- A subgroup analysis based on certain patient characteristics will help to determine the incidence for specific populations and screen the populations with higher than average risk of delirium.

- An understanding of the risk factors that predict delirium will contribute to the early recognition of delirium and the formulation of prevention strategies, resulting to reduce postoperative delirium and its associated effects.

- The findings of this study will help guide clinicians to care the patients with AMI undergoing interventional therapy more efficiently, and researchers as they develop intervention trials to prevent delirium in this potentially vulnerable population.

- Only English articles will be included in our study and this may cause publication bias to some extent.

\section{INTRODUCTION}

Delirium is a disorder hallmarked by an acute disturbance in attention and cognition. Common characteristics of delirium include inability to direct, focus, sustain, or shift attention, and cognitive disturbances include impairment of memory, orientation, perception and consciousness. ${ }^{12}$ Delirium in the postoperative period is a wide-reaching problem that affects important clinical outcomes. Multiple studies have found significant associations between development of postoperative delirium and longer hospital stay, higher care cost, increased hospital readmission rates, as well as a prolonged cognitive 
impairment and poorer social functioning. ${ }^{3-5}$ In addition, patients who experience postoperative delirium also have an increased mortality. ${ }^{67}$

Coronary artery disease is the one of the most common causes of death all over the world. ${ }^{8}$ Coronary artery bypass graft surgery and percutaneous coronary intervention (PCI) are two mainly therapy of coronary artery disease. For patients undergo coronary artery bypass graft surgery, postoperative delirium has been investigated extensively. Previous studies have confirmed that delirium after coronary artery bypass graft surgery is common and associated with adverse outcomes. ${ }^{5-11}$ Acute myocardial infarction (AMI) is one of the severe manifestations of coronary artery disease. PCI has become increasingly important and common in the treatment of AMI, resulting in improving coronary perfusion, fewer ischaemic complications, more survival myocytes, preserved ventricular function and improved survival of patients. ${ }^{12} 13$ The incidence and risk factors of delirium in individuals with AMI after primary PCI has not been completely determined and no relevant systematic review and meta-analysis of incidence or risk factors exists. Such a systematic review and meta-analysis is needed for several reasons. Future intervention studies aimed at preventing delirium will need an accurate estimate of incidence, which could be used to help researchers perform sample size and power calculations for trials. In addition, the incidence of postoperative delirium may vary depending on patient characteristics and operation correlative factors. A systematic review and meta-analysis that analyses and identifies higher incidence of certain subgroups could help future researchers focus interventions on these high-risk populations. Finally, there are few effective treatments for established delirium, but postoperative delirium and its associated effects can be reduced through prevention strategies and early recognition. ${ }^{14}$ Prevention strategies require an understanding of the risk factors that predict delirium. Some studies have explored the risk factors of delirium in other populations, ${ }^{15-17}$ but for patients being diagnosed AMI and undergoing PCI, it still need to be further analysed and ummarised.

Hence, we intend to conduct a systematic review and meta-analysis to ascertain the incidence of delirium among AMI patients undergoing PCI. We will also ascertain incidence based on the patient characteristics that may have a higher than average expected incidence. It has been confirmed that there are numerous patient characteristics or operation correlative factors associated with delirium. ${ }^{18-21}$ We will conduct a subgroup analysis mainly based on these characteristics and operation correlative factors, including, but not limited to, age over 65 years, diabetes mellitus, cerebrovascular disease and depression, duration of operation, intraoperative hypotension, as well as the degree of coronary artery lesions. Moreover, another aim of this systematic review is to identify key risk factors for delirium in AMI patients undergoing PCI.

\section{METHODS AND ANALYSIS}

Prior to the start of the project, a search in the relevant databases showed that there was no previous or ongoing systematic review on this subject. The article will be reported following the recommendations of the Preferred Reporting Items for Systematic Review and Meta-Analysis Protocols, ${ }^{22}$ and of meta-analyses of observational studies. ${ }^{23}$ The study has been registered on the International Prospective Register of Systematic Reviews database (PROSPERO) on 5 May 2020. The study is expected to start on 1 October 2020 and be completed on 31 December 2021. Should any amendments to this protocol be necessary, they will be recorded on the PROSPERO platform.

\section{Information sources}

Multiple sources will be used in this systematic review. A systematic comprehensive search will be performed in the following online databases: PubMed, EMBASE, Cochrane Library, PsycINFO, CINAHL and Google Scholar. Bibliographies of all included studies and any other relevant reviews or meta-analyses identified via the search will be screened. All databases will be searched from their inception to the search date.

\section{Search strategy}

The search terms are combinations of myocardial infarction, ischaemic heart disease, acute coronary syndrome, PCI, delirium, acute confusion, acute organic psychosyndrome (see the search strategy in online supplemental appendix 1).

\section{Eligibility criteria}

We will consider the following criteria for study inclusion: (1) full-length reports published in peer-reviewed journals; (2) prospective cohort studies or cross-sectional studies on patients who was diagnosed AMI and underwent coronary interventional therapy; (3) studies in which a validated measurement tool was used to screen for delirium as well as those that used diagnostic criteria for delirium as described in the Diagnostic and Statistical Manual of Mental Disorders or International Classification of Diseases, such as the Confusion Assessment Method (CAM),${ }^{24}$ Delirium Rating Scale,${ }^{25}$ CAM for the Intensive Care Unit, ${ }^{26}$ Intensive Care Delirium Screening Checklist $^{27}$ and so on; (4) at least incidence reported or one risk factor identified as being associated with delirium. If the inclusion criteria are not met, the study will be excluded.

\section{Outcome}

The primary outcome is the incidence of postoperative delirium, which is diagnosed using a validated delirium assessment tool and defined as an episode of delirium occurring after PCI.

\section{Study selection and data extraction}

All records identified in the databased will be collected in the reference management software EndNote X8 
(Thomson Reuters, New York, New York, USA), and deduplication will be performed. On deduplication, records will be screened in two stages. First, the title and the abstract of all records will be screened against the aforementioned eligibility criteria. Second, full texts of all articles not excluded in the first stage will be reviewed to determine whether all relevant criteria are met. Both stages will be performed independently by two reviewers. Disagreement will be resolved by consensus. If no consensus can be reached, disagreement will be resolved by adjudication of a designated third reviewer. We will present the process of search and study selection using a flow process chart. The general features cover the title, first author, publication year, country, study type, sample size, patient characteristics, patients who underwent interventional therapy, delirium diagnosis tool, incidence duration of delirium and significant factors.

\section{Quality assessment}

Two reviewers will independently evaluate the quality of the studies, and the level of agreement between them will be recorded. Any disagreements between the two reviewers will be resolved by discussion with a third reviewer. The risk of bias tool for prevalence studies developed by Hoy et al, and the Cochrane guidelines ${ }^{2328}$ will be used to assess the quality of included studies. Risk of bias and quality scores will be presented in a table.

\section{Statistical analysis}

The meta-analysis of comparable data will be performed using Stata V.15.0 software. An overall summary estimate of the incidence will be calculated from studies reporting incidence of delirium among AMI patients undergoing PCI. Inverse variance method or generalised linear mixed model may be used for pooling. The pooled incidence with $95 \%$ CI will be given for either the fixed-effect model or the random-effects model. Statistical heterogeneity across studies will be assessed by the Cochrane $\mathrm{Q}$ test and quantified by calculating $\mathrm{I}^{2}$. An $\mathrm{I}^{2}$ of $>50 \%$ will be considered to indicate substantial heterogeneity. The random-effects model or the fixed-effect model will be used depending on the heterogeneity of studies included. Where heterogeneity is statistically significant, we will conduct a subgroup analysis to investigate the possible sources of heterogeneity according to the patient characteristics and operation correlative factors, such as age over 65 years, diabetes mellitus, cerebrovascular disease and depression, diagnostic methods, duration of operation, intraoperative hypotension and the degree of coronary artery lesions. Furthermore, a sensitivity analysis will be performed to find out how our results would change if only high-quality studies were considered.

To analyse associations with postoperative delirium, each risk factor reported in the included studies will be recorded together with its statistical significance; narrative (tabular) and meta-analytical approaches (forest plots) will be employed. Univariable risk factors will be summarised for each included study. Pooled measure will be calculated as the inverse variance weighted mean of the OR for categorical data, or mean difference for continuous data with $95 \% \mathrm{CI}$ to assess the strength of association between the risk factors and postoperative delirium. Statistical heterogeneity will also be assessed. A randomeffects model will be used for heterogeneous data. Otherwise, a fixed-effect model will be used. Meta-regression will be performed to assess the potentially important covariates that might exert substantial impact on between study heterogeneity. A funnel plot and Egger test will be used to assess the potential for publication bias.

\section{Patient and public involvement \\ No patient involved.}

\section{DISCUSSION}

This systematic review will provide an overview of the current state of evidence concerning the incidence and risk factors for delirium after PCI in the patients with AMI. To the best of our knowledge, this will be the first systematic review concerning this topic. The primary outcomes analysed will provide evidence for clinicians to attach importance to the current burden of postoperative delirium in the patients with AMI undergoing PCI, and providing more care to this potentially vulnerable population. Secondary, outcomes will provide information to help guide researchers as they develop interventional trials to prevent this important syndrome in this high-risk population. Furthermore, gaps in the current literature will be identified, and recommendations for future avenues of research will be given. Strengths of this systematic review include the search in multiple databases, the systematic approach including screening, data extraction and quality assessment by two independent reviewers, as well as transparency in reporting according to guidelines. The main limitation is the language restriction to English. Only English articles will be included in our study, resulting to the fact that relevant data from non-English spoken areas may lose, and this may cause publication bias to some extent.

Contributors $\mathrm{KH}$ and JL conceived and designed the protocol. KH and SL drafted the protocol manuscript. HY, TC and XQ critically revised the manuscript for methodological and intellectual content. DD and YZ participated in the development of the search strategy. HP and XW planned the data extraction. All authors approved the final version.

Funding The authors have not declared a specific grant for this research from any funding agency in the public, commercial or not-for-profit sectors.

Competing interests None declared.

Patient consent for publication Not required.

Provenance and peer review Not commissioned; externally peer reviewed.

Supplemental material This content has been supplied by the author(s). It has not been vetted by BMJ Publishing Group Limited (BMJ) and may not have been peer-reviewed. Any opinions or recommendations discussed are solely those of the author(s) and are not endorsed by BMJ. BMJ disclaims all liability and responsibility arising from any reliance placed on the content. Where the content includes any translated material, BMJ does not warrant the accuracy and reliability of the translations (including but not limited to local regulations, clinical guidelines, 
terminology, drug names and drug dosages), and is not responsible for any error and/or omissions arising from translation and adaptation or otherwise.

Open access This is an open access article distributed in accordance with the Creative Commons Attribution Non Commercial (CC BY-NC 4.0) license, which permits others to distribute, remix, adapt, build upon this work non-commercially, and license their derivative works on different terms, provided the original work is properly cited, appropriate credit is given, any changes made indicated, and the use is non-commercial. See: http://creativecommons.org/licenses/by-nc/4.0/.

\section{ORCID iD}

Kaizhuang Huang http://orcid.org/0000-0002-2740-1387

\section{REFERENCES}

1 Rengel KF, Pandharipande PP, Hughes CG. Postoperative delirium Presse Med 2018;47:e53-64.

2 Saczynski JS, Marcantonio ER, Quach L, et al. Cognitive trajectories after postoperative delirium. N Engl J Med 2012;367:30-9.

3 Neufeld KJ, Leoutsakos J-MS, Sieber FE, et al. Outcomes of early delirium diagnosis after general anesthesia in the elderly. Anesth Analg 2013;117:471-8.

4 Slooter AJC, Van De Leur RR, Zaal IJ. Delirium in critically ill patients. Handb Clin Neurol 2017;141:449-66.

5 Lingehall HC, Smulter NS, Lindahl E, et al. Preoperative cognitive performance and postoperative delirium are independently associated with future dementia in older people who have undergone cardiac surgery: a longitudinal cohort study. Crit Care Med 2017;45:1295-303.

6 Moskowitz EE, Overbey DM, Jones TS, et al. Post-operative delirium is associated with increased 5-year mortality. Am J Surg 2017;214:1036-8.

7 Witlox J, Eurelings LSM, de Jonghe JFM, et al. Delirium in elderly patients and the risk of postdischarge mortality, institutionalization, and dementia: a meta-analysis. JAMA 2010;304:443-51.

8 Dalen JE, Alpert JS, Goldberg RJ, et al. The epidemic of the 20(th) century: coronary heart disease. Am J Med 2014;127:807-12.

9 Li H-C, Chen Y-S, Chiu M-J, et al. Delirium, subsyndromal delirium, and cognitive changes in individuals undergoing elective coronary artery bypass graft surgery. J Cardiovasc Nurs 2015;30:340-5.

10 Oldham MA, Hawkins KA, Yuh DD, et al. Cognitive and functional status predictors of delirium and delirium severity after coronary artery bypass graft surgery: an interim analysis of the neuropsychiatric outcomes after heart surgery study. Int Psychogeriatr 2015;27:1929-38.

11 Gottesman RF, Grega MA, Bailey MM, et al. Delirium after coronary artery bypass graft surgery and late mortality. Ann Neurol 2010;67:338-44.

12 Marenzi G, De Metrio M, Rubino M, et al. Acute hyperglycemia and contrast-induced nephropathy in primary percutaneous coronary intervention. Am Heart J 2010;160:1170-7.
13 Aversano T, Aversano LT, Passamani E, et al. Thrombolytic therapy vs primary percutaneous coronary intervention for myocardial infarction in patients presenting to hospitals without on-site cardiac surgery: a randomized controlled trial. JAMA 2002;287:1943-51.

14 Trzepacz PT, Mittal D, Torres R, et al. Validation of the delirium rating Scale-revised-98: comparison with the delirium rating scale and the cognitive test for delirium. J Neuropsychiatry Clin Neurosci 2001;13:229-42.

15 Tomlinson EJ, Phillips NM, Mohebbi M, et al. Risk factors for incident delirium in an acute general medical setting: a retrospective casecontrol study. J Clin Nurs 2017;26:658-67.

16 Kubota K, Suzuki A, Onde S, et al. Age is the most significantly associated risk factor with the development of delirium in patients hospitalized for more than five days in surgical wards: retrospective cohort study. Ann Surg 2018;267:874-7.

17 Janssen TL, Steyerberg EW, Faes MC, et al. Risk factors for postoperative delirium after elective major abdominal surgery in elderly patients: a cohort study. Int J Surg 2019;71:29-35.

18 Zaal IJ, Devlin JW, Peelen LM, et al. A systematic review of risk factors for delirium in the ICU. Crit Care Med 2015;43:40-7.

19 Bilotta F, Lauretta MP, Borozdina A, et al. Postoperative delirium: risk factors, diagnosis and perioperative care. Minerva Anestesiol 2013;79:1066-76.

20 Gosselt AN, Slooter AJ, Boere PR, et al. Risk factors for delirium after on-pump cardiac surgery: a systematic review. Crit Care 2015;19:346.

$21 \mathrm{Mu}$ JL, Lee A, Joynt GM. Pharmacologic agents for the prevention and treatment of delirium in patients undergoing cardiac surgery: systematic review and metaanalysis. Crit Care Med 2015;43:194-204.

22 Moher D, Shamseer L, Clarke M, et al. Preferred reporting items for systematic review and meta-analysis protocols (PRISMA-P) 2015 statement. Syst Rev 2015;4:1.

23 Stroup DF, Berlin JA, Morton SC, et al. Meta-Analysis of observational studies in epidemiology: a proposal for reporting. meta-analysis of observational studies in epidemiology (moose) group. JAMA 2000;283:2008-12.

24 Inouye SK, van Dyck CH, Alessi CA, et al. Clarifying confusion: the confusion assessment method. A new method for detection of delirium. Ann Intern Med 1990;113:941-8.

25 Trzepacz PT, Baker RW, Greenhouse J. A symptom rating scale for delirium. Psychiatry Res 1988;23:89-97.

26 Ely EW, Inouye SK, Bernard GR, et al. Delirium in mechanically ventilated patients: validity and reliability of the confusion assessment method for the intensive care unit (CAM-ICU). JAMA 2001;286:2703-10.

27 Bergeron N, Dubois MJ, Dumont M, et al. Intensive care delirium screening checklist: evaluation of a new screening tool. Intensive Care Med 2001;27:859-64.

28 Hoy D, Brooks P, Woolf A, et al. Assessing risk of bias in prevalence studies: modification of an existing tool and evidence of interrater agreement. J Clin Epidemiol 2012;65:934-9. 